\title{
Magnesium Functions as Superior Co-factor for Measuring Reverse Transcriptase Activity of HIV-1, HIV- 2, and SIV
}

\author{
Salequl Islam, Mohammad Ali Moni, Atsushi Tanaka, and Hiroo Hoshino
}

\begin{abstract}
This study compared different detection methods of human/simian immunodeficiency virus (HIV/SIV) infections in the cell line systems; notably, i) Indirect immunofluorescence assay (IFA), ii) integrated proviral DNA detection, iii) detection of syncytia, iv) measurement of reverse transcriptase (RT) activity. RTs of various retroviruses require cations, including $\mathrm{Mg}^{2+}, \mathrm{Mn}^{2+}, \mathrm{Ni}^{2+}$, and $\mathrm{Cu}^{2+}$, for their enzyme-activities. The study further compared the roles of $\mathrm{Mg}^{2+}$ and $\mathrm{Mn}^{2+}$ as cofactors for RT activities of freshly harvested HIV-1, HIV-2, and SIV. The NP-2/CD4/coreceptor cells were seeded for overnight and infected with viral inoculums at a multiplicity of infection (MOI) 1.0. The cells were passaged regularly in a 2-3 days interval and maintained up to 2 weeks. Infected cells were detected by indirect immunofluorescence assay (IFA). Multinucleated giant cells (MGC) in syncytia were quantified by Giemsa-staining. Proviral DNA was detected by PCR, and reverse transcriptase (RT) activity was measured. Two different cations, Mg2+ and $\mathrm{Mn}^{2+}$ were used as cofactors for RT assay. We found all the strains of HIV-1, HV-2 and SIV to infection in the cell line conveniently. IFA had identified all the viral infections in the infected cells. Proviral DNA detection, syncytia formation was observed in the infected cells. We found a better performance of $\mathrm{Mg2}+$ as cofactor over $\mathrm{Mn}^{2+}$ in RT assay for HIV-1, HIV-2, SIV. Different four detection techniques of HIV/SIV infections show high level of agreement in the NP-2-based cell line system. $\mathrm{Mg}^{2+}$ remains a better cofactor for $\mathrm{RT}$.
\end{abstract}

Index Terms - HIV/SIV, RT assay, IFA, Proviral DNA, Syncytia.

\section{INTRODUCTION}

Both primary cell cultures and continuous cell lines function as fundamental tools for basic research in numerous life science, particularly where in-vivo experiments are challenging. Cell lines have been used for several decades to investigate different human pathogenic viruses like human immunodeficiency virus type 1 (HIV-1), human T-cell leukemia/lymphoma virus type I and II (HTLV-I and -II), and hepatitis viruses. Different cell lines were established from patient material linked to several

Published on August 23, 2020.

Salequl Islam, Department of Microbiology, Jahangirnagar University, Bangladesh.

(corresponding e-mail: salequl@juniv.edu)

Mohammad Ali Moni, School of Public Health and Community Medicine, UNSW Sydney, Australia.

(e-mail: m.moni@unsw.edu.au)

Atsushi Tanaka, Gunma University Graduate School of Medicine, Japan.

(e-mail: washi69@biken.osaka-u.ac.jp).

Hiroo Hoshino, Gunma University Graduate School of Medicine, Japan. (e-mail: hoshino@ ${ }^{\circledR}$ unma-u.ac.jp). virus-specific tumors and cancers [1], and some cell lines are known to retain human pathogenic viruses inherently, for example, well-known HeLa cell line harbors the human papillomavirus integrated into its genome as a provirus [2]. The human glioma cell line, NP-2 is a well-known cellsystem for receptor/coreceptor evaluation study for Human and Simian Immunodeficiency viruses (HIV and SIV) [3]. HIV and SIV gain access to susceptible cells by using CD4 as a receptor [4], [5] and chemokine receptors (CKRs), CCR5 and/or CXCR4, as their major coreceptors [6], [7]. Established cell lines, NP-2/CD4/CCR5 and NP2/CD4/CXCR4 were reported as HIV/SIV-susceptible host cells carrying both receptor (CD4) and any of major coreceptor (CCR5/CXCR4). In contrast, NP-2/CD4 and NP2 were used as HIV/SIV-resistant model cell lines lacking the receptor or any coreceptor [3]. Several methods generally make the assessment of HIV/SIV infection in the cell line models. Immunofluorescence assay (IFA) is a technique that has been used to detect retroviral antigens, including HIV/SIV in the infected cells [8], [9]. IFA allows discrimination between viral-infected and -uninfected cells. Immunofluorescence is a recognized and popular assay for viral antigen detection in clinical samples and antibody measurement in serum [10]. The presence of HIV can be further confirmed by detecting the proviral DNA integration into host cellular DNA [11].

Productive retroviral infection in susceptible cells in vitro or in vivo is often found with the formation of syncytia, which are multinucleated giant cells (MGC) [12], [13]. Progressive HIV infection induces more syncytia formation, and ultimately the cells in the culture die as a consequence of the virus-induced cytopathic effect [14]. Cells rupture and viruses release into the culture supernatant. HIV and other retroviruses possess reverse transcriptase (RT) enzyme bearing two major enzymatic activities: A DNA polymerase activity (complementary DNA synthesis) and a nuclease activity, termed ribonuclease $\mathrm{H}$ (RNase $\mathrm{H}$, that degrades RNA which is part of an RNA-DNA hybrid). RT activity remains a recognized tool for the detection of retroviruses [15]. Therefore, the presence of HIV in the culture supernatant fluid can be detected by RT activity levels. The viral recovery by RT assay is validated well in AIDS patients as well [16]. Both cDNA synthesis activity and ribonuclease $\mathrm{H}$ activity of $\mathrm{RT}$ require a divalent cation as an essential cofactor [17], [18]. Retroviruses from various origins are genetically divergent and structurally distinct, but that share similar cation requirements for their RT activity, including $\mathrm{Mg}^{2+}, \mathrm{Mn}^{2+}, \mathrm{Ni}^{2+}$, and $\mathrm{Cu}^{2+}$ [19]. The presence of $\mathrm{Zn}^{2+}$ was found to form a highly stable RT with 
much reduced catalytic activity [20]. $\mathrm{Mg}^{2+}$ was reported as improved cofactor RT activities for some simian immunodeficiency viruses [21], but not for HIV-1 and HIV2. We designed this study to compare different detection methods of HIV infections in cell lines. Further, the study compared the roles of two divalent cations, $\mathrm{Mg}^{2+}$ and $\mathrm{Mn}^{2+}$ as cofactors in measuring RT activities in freshly harvested HIV-1, HIV-2, and SIV in the cell culture spent media.

\section{MATERIALS AND MEthODS}

A. Amplification and Cloning of HIV-Receptor and Coreceptors

The coding region DNA sequences of CD4, CCR5, and CXCR4 were obtained from the GenBank database (Accession number, M12807.1, U54994.1, and AY242129.1, respectively). As a control, the mRNA expression of gleceraldehyde-3-phospahte dehydrogenase (GAPDH, GenBank accession number M17851.1) was also examined. Oligonucleotide primers were designed and synthesized (Proligo K.K., Tokyo, Japan) covering their open reading frames (ORFs) to detect reverse-transcribed mRNAs by polymerase chain reaction (PCR). The ORFs amplified by RT-PCR were detected by electrophoresis through $1 \%(\mathrm{w} / \mathrm{v})$ agarose gel. Amplified ORFs were cloned into a TA-cloning plasmid, pGEM-T Easy (Promega, Madison, WI) one by one, and the sequencing of each cloned DNA fragment was performed by a 5500-sequencer (Hitachi, Tokyo, Japan) using fluorescent primers labeled with Texas Red. Restriction-digested each ORF was recloned into an expression plasmid for transfected into recipient NP-2 cells.

\section{B. Establishment of Cell System for viral propagation}

As HIV/SIV-susceptible host cells, NP-2/CD4/CCR5 and NP-2/CD4/CXCR4 were established [3]. Both NP-2/CD4 and NP-2 cell lines were used as HIV/SIV-resistant negative control host systems. To establish NP-2/CD4/CCR5 and NP-2/CD4/CXCR4 cell lines, we used the retroviral expression plasmid pMX-puro that harbors an SV40 promoter-controlled puromycin-resistant gene between the multicloning sites and the 3 '-long terminal repeat (LTR) of Moloney murine leukemia virus [22]. NP-2 cells were transfected with expression plasmids for CD4 receptor and CCR5/CXCR4 coreceptor candidates to generate NP2/CD4/Coreceptor cells. According to the manufacturer's protocol, transfection was performed using FuGENE 6 (Roche, Basel, Switzerland). Transfected cells were selected by maintaining these cells in culture medium containing one $\mu \mathrm{g} / \mathrm{ml}$ puromycin for 2-3 weeks as described elsewhere [23]. RT-PCR examined the expression of CD4, CCR5, and CXCR4 in these cells. For this, total RNA was isolated, and reverse-transcribed using the same PCR primers used to clone the candidate genes. As controls, GAPDH mRNA in each cDNA preparation was detected. Eagle's minimum essential medium (EMEM) (NISSUI Co., Inc.) with $10 \%$ FBS was used to maintain the NP-2 and its derivatives expressing CD4 and CCR5/CXCR4.

In parallel, a human T-cell line, C8166, expressing the CD4 receptor and CXCR4 coreceptor, and the C8166 cell line transduced with CCR5, C8166/CCR5, were used for the preparation of viral stocks of HIV-1, HIV-2 and SIV strains.
These cells were maintained in RPMI 1640 medium containing 10\% FBS (NISSUI Co. Inc., Japan).

\section{Viruses}

Both laboratory-adapted strains and primary isolates of HIV-1, HIV-2, and SIV were utilized to check their infection dynamics and reverse transcriptase activities. The established cell line-adapted CCR5 tropic HIV-1 strains BaL [24], and CXCR4-tropic HIV-1 strain IIIB [25] were used. Both the HIV-1 strains were classified as subtypes B [26] based on their envelope amino acid sequences. As for HIV-2 strain, ROD [27], and SIV strain, mac251 [28] were used. One HIV-2 and one SIV were obtained from the National Institute for Biological Standard and Control (NIBSC, London, UK), were also used. The primary HIV-2 (MIR, NIBSC reference EVA171) was collected from a patient of Guinea Bissau. The primary SIV (smE660, NIBSC reference ARP1040) was originated from a sooty mangabey.

\section{HIV/SIV Infection Assay}

NP-2/CD4/CCR5, NP-2/CD4/CXCR4, and NP-2/CD4 cells were seeded into 24 -well plates at a number of $2.5 \times 10^{4}$ cells/well. The next day, the medium was drained off with the addition of viral inoculums at a multiplicity of infection (MOI) 1.0 to the seeded-cells. Following a six-hour incubation, the cells were washed three times with EMEM containing $10 \%$ FBS to remove free viruses, refilled finally with $500 \mu \mathrm{L}$ fresh medium per well, and cultured at $37^{\circ} \mathrm{C}$ in $5 \% \mathrm{CO} 2$ incubator. The cells were passaged regularly in a $2-$ 3 days interval and maintained up to 2 weeks.

\section{E. Indirect Immunofluorescence Assay (IFA)}

The presence of viral antigens in the infected cells was detected by indirect immunofluorescence assay (IFA) [29]. Briefly, virus-infected cells were used as a source of antigens. Uninfected cells were included on the slides in parallel as a control-control for specificity. Cells were smeared for IFA onto glass slides in duplicate, air-dried, fixed with acetone. Pooled anti-HIV human sera were diluted 1:10 in phosphate-buffered saline (PBS) and used as a primary antibody to detect antigen of HIV-1 and HIV-2. Similarly, anti-SIV monkey serum was used to detect SIVsmE660- and SIVmac251-antigens. The cells were then incubated for $30 \mathrm{~min}$ at $37{ }^{\circ} \mathrm{C}$ in humid condition. After washing three times with PBS and them dried, fluorescein isothiocyanate (FITC)-conjugated goat anti-human and antimonkey IgG were used as secondary antibodies (1:200 dilution, $10 \mathrm{uL}$ ) was applied to each well and incubated at $37{ }^{\circ} \mathrm{C}$ in a humidity box for $30 \mathrm{~min}$ followed by washing as described. The solution of the buffered glycerol ( $\mathrm{pH}$ : 8.0) was placed on the slides, followed by a coverslip. Fluorescence in antigen-positive cells was observed under UV light in a specialized microscope.

\section{F. Detection of Proviral DNA and Syncytia}

HIV/SIV infection was further assured by the detection of proviral DNA by PCR using the genomic DNA of infected cells as templates. HIV-1 and HIV-2 primers were designed covering conserved gag-region gene, and SIV primer was designed targeting env-region gene. Infection induced cell fusion was observed under light microscopy. The formation of syncytia was detected by Giemsa staining (Muto Pure 
Chemicals, Tokyo, Japan).

\section{G. RT assay}

Free viruses in the spent culture supernatants were quantified by measuring reverse transcriptase (RT) activities. RT is an enzyme unique to a group of viruses known as retroviruses, of which the HIV/SIV are members. There are several published RT assay procedures for those laboratories interested in detecting RT activity in concentrated culture supernatant fluid [30]-[32]. The principle of most of these RT assays is as follows. In the RT assay, an RNA template primer, (oligodeoxythymidylate 1218, known as oligo dT12-18) is mixed with radioactive tritium, $[3 \mathrm{H}]$ thymidine, a buffer containing dithiothreitol as a reductant, Triton $\mathrm{X}-100$ as a detergent to disrupt whole virus particles, and a sample of concentrated culture supernatant. The reaction buffer also requires a di-ionic cation as a cofactor for RT enzymes. This study has examined the comparative cofactor roles $\mathrm{Mg}^{2+}$ and $\mathrm{Mn} 2+$ in RT activities. The complete reaction-mixture was incubated at $37^{\circ} \mathrm{C}$ for one hour. In the presence of RT, the radioactive thymidine should be incorporated onto the template. Cold acid was added to stop the reaction and precipitate the strands. After an incubation on ice, the reaction mixture was poured over a blotting paper to trap the strands and washed to remove unbound $[3 \mathrm{H}]$ thymidine. The blotting papers were put into scintillation vials, dried, and counted in scintillation cocktail. Activity (in counts per minute, cpm) over a specific limit was indicative of HIV/SIV presence in the sample. The mean cpm values of the duplicate samples were determined.

\section{RESULTS}

\section{A. Establishment of NP-2/CD4/Coreceptor cell systems}

Amplified ORFs of CD4, CCR5, and CXCR4 were transduced into NP-2 cells to produce NP-2/CD4/Coreceptor cells. The expressions of the receptor/coreceptor were affirmed by detecting their specific mRNAs in the transfected cell lines by RT-PCR. We reassured the expressions of CD4, CCR5, and CXCR4 in NP2/CD4/CCR5 and NP-2/CD4/CXCR4 cell-lines by flow cytometry (FCM).

\section{B. Detection of viral infection by IFA, Cell-cytopathy, and Proviral DNA}

In this study, a total of six HIV/SIV infections were inoculated in NP-2/CD4/coreceptor cells separately. Viral antigens were confirmed in the infected cells by IFA. Both NP-2 and NP-2/CD4 cells appeared antigen-negative in all the viral infections. Therefore, the overall sensitivity and specificity of IFA were $100 \%$ for the HIV/SIV antigen detection in the cell-line system described here. The appearance of antigen-positive and antigen-negative cells by IFA was shown in Fig. 1 a. All the HIV-1 and HIV-2 showed rapid infectivity through CCR5 and CXCR4 coreceptors and took 5-10 days after inoculation to make about $80-90 \%$ NP-2/CD4/coreceptor cells antigen-positive. SIVmac251 was found to infect NP-2/CD4/CCR5 cells comparative slowly and took two weeks to reach $80-90 \%$ cells antigen-positive by IFA. However, SIVsm660 was a rapid grower. Structural changes of virus-infected NP2/CD4/CCR5 and NP-2/CD4/CXCR4 cells as the ballooning of fused cells were detected under a light microscope as the sign of cytopathic effect (Fig. 1 b). Dual and CXCR4-tropic viruses formed more CPE than CCR5-tropic viruses. SIVmac251 did not show CPE in cells until the end of the assay went for about two weeks when majority cells become antigen-positive by IFA. Cytopathic effects of HIV/SIV infected cells showed harmony of syncytia formation through CCR5/CXCR4-transduced NP-2/CD4 cells. We detected clusters of multinucleated giant cells (MGC) in syncytia after fixation and staining with Giemsa (Fig. 1 c). Likely, dual and X4-tropic viruses were found as more syncytia-inducer. MGC clusters were variable in sizes and consisting of 10-20 nuclei per syncytium. Receptor/coreceptor-negative NP-2 and NP-2/CD4 cells did not form any cluster of nuclei. When cells become infected by HIV/SIV, provirus DNA is supposed to form by reverse transcription of genomic RNA into double-stranded DNA followed by the subsequent integration process. We further validated infection through NP-2/CD4/coreceptor cells by detecting proviral DNA in infected cell lines (Fig. $1 \mathrm{~d}$ ).

\section{Detection of viral infection by RT assay and cofactor effects}

Cell bursting after cytopathic effects in the cell-linesystem released a large amount of the viruses in the supernatants. The amount of liberated virus was assessed by reverse transcriptase activities in spent culture media of viral inoculated cells. For the RT assay, culture fluids of different viruses were collected when $70-90 \%$ cells were viral antigen-positive by IFA. The RT activities of HIV-1BaL using CCR5 was $2.3 \times 10^{5} \mathrm{cpm} / \mathrm{mL}$ when $\mathrm{Mg}^{2+}$ was used as a cofactor and $7.4 \times 10^{4}$ when $\mathrm{Mn}^{2+}$ was used as a cofactor. CXCR4-tropic HIV-1IIIB also generated higher RT activity, $2.3 \times 10^{5} \mathrm{cpm} / \mathrm{mL}$ with $\mathrm{Mg}^{2+}$, and $1.1 \times 10^{5} \mathrm{cpm} / \mathrm{mL}$ with $\mathrm{Mn}^{2+}$. Therefore, regardless of coreceptor tropism, $\mathrm{Mg}^{2+}$ functioned as better cofactors for HIV-1 RT. Replacement of $\mathrm{Mg}^{2+}$ by $\mathrm{Mn}^{2+}$ has made two-three-fold reduced RT activities. Similar results were found for all the remaining HIV-2, and SIV tested in this study. Very low RT activities were detected when the cell culture supernatants without virus inoculum were measured (Fig. 2). For all the HIV/SIV, the detection of RT activities shows good harmony with IFA, CPE, and proviral DNA amplification (Table 1).

TABLE 1: COMPARATIVE DETECTION OF HIV-1, HIV-2, AND SIV INFECTIONS IN NP-2/CD4/CORECEPTOR CELL LINES BY DIFFERENT METHODS

\begin{tabular}{|c|c|c|c|c|c|}
\hline \multicolumn{2}{|c|}{ Virus (major coreceptor tropism) } & \multicolumn{4}{|c|}{ Infection in NP -2/CD4/coreceptor ${ }^{b}$ cells by: } \\
\hline & & \multirow{2}{*}{$\begin{array}{l}\text { IFA }^{\mathrm{c}} \\
+++\end{array}$} & \multirow{2}{*}{$\begin{array}{c}\begin{array}{c}\text { Proviral } \\
\text { DNA }\end{array} \\
++\end{array}$} & \multirow{2}{*}{$\begin{array}{c}\begin{array}{c}\text { Syncytia } \\
\text { format ion } \mathbf{d}\end{array} \\
++\end{array}$} & \multirow{2}{*}{$\frac{\text { RT activity }}{++}$} \\
\hline HIV-1 & IIIB (X4) & & & & \\
\hline & $\mathrm{BaL}(\mathrm{R} 5)$ & +++ & ++ & + & ++ \\
\hline \multirow[t]{2}{*}{ HIV -2} & ROD (R5X4) & +++ & ++ & ++ & ++ \\
\hline & MIR (R5X4) & +++ & ++ & ++ & ++ \\
\hline \multirow[t]{2}{*}{ SIV } & Mac251 (R5) & +++ & ++ & - & ++ \\
\hline & smE660 (R5) & +++ & ++ & + & +++ \\
\hline
\end{tabular}

a Major coreceptor tropism, R5=CCR5-tropic, X4=CXCR4 tropic, and R5X4=dual tropic.

b HIV-1 IIB was propagated in NP-2/CD4/CXCR4 cells and the remaining viruses were grown in NP-2/CD4/CCR5 cells.

c Viral antigen positive cells were examined by indirect immunofluorescence assay (IFA).,$+++ 70-90 \%$ of cells became antigen- 
positive within 10 days of infection.

d Syncytia formation was more common in CXCR4 tropic and dual tropic viruses.

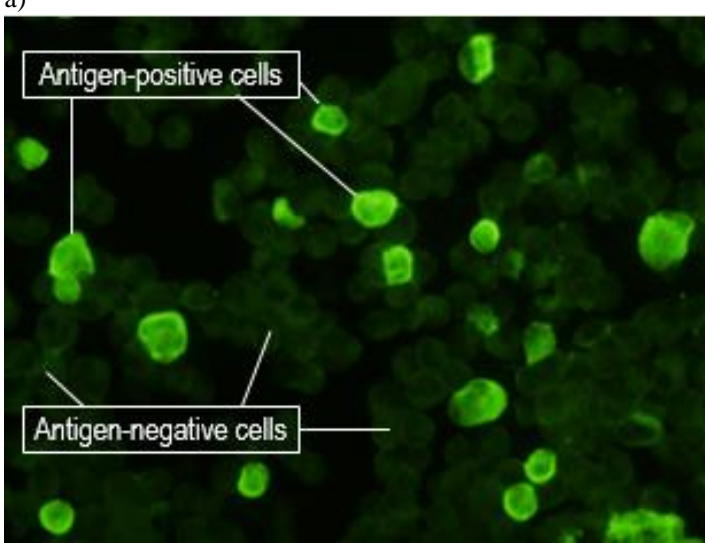

Indirect Immunoflurescence assay (IFA)

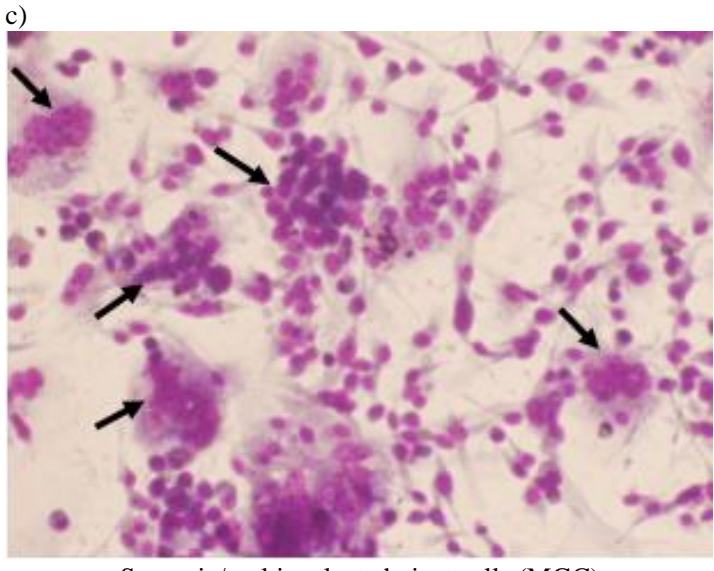

Syncytia/multinucleated giant cells (MGC) e Primary isolate SIVsmE660 produced extraordinarily high RT activities in multiple independent experiments.

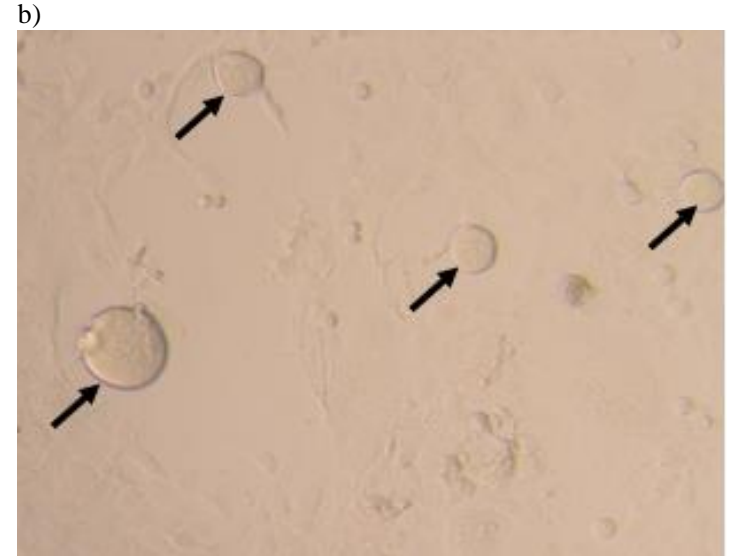

Retroviral cytopathic effects (CPE)

d)

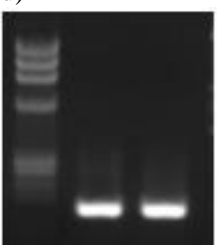

HIV-1, gag

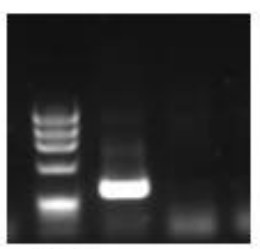

HIV-2, gag

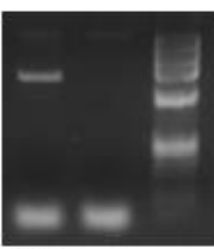

SIV, env

Amplified Proviral DNA

Fig. 1. Detection of HIV/SIV infections in NP-2/CD4/coreceptor cells. a) The NP-2/CD4/coreceptor cells were seeded separately for each of HIV/SIV, and viruses were inoculated the next day. Cells were passaged in 2-3 days interval and continued for two weeks. Viral antigens in infected cells were determined by indirect immunofluorescence assay (IFA), for which cells were smeared onto glass slides in duplicate, dried, fixed by acetone, and stained by pooled human anti-HIV sera for HIV-1 and HIV-2 and by monkey anti-SIV serum for SIV. Fluorescein isothiocyanate (FITC)-conjugated goat anti-human and anti-monkey IgG were used as secondary antibodies. Cells were visualized under a UV fluorescence microscope through FITC-specific filter. b) The ballooning of infected cells manifested cytopathic effects (CPE) of HIV/SIV as a sign of acute infection. The arrows indicated cells demonstrating CPE. c) Virally infected cells were characterized by multinucleated giant cells that were detected by Giemsa staining. NP-2/CD4/CCR5 and NP-2/CD4/CXCR4 cells were cultured with viral inoculums for 3-10 days and then prepared smear for Giemsa staining. The arrows indicated multinucleated syncytia that appeared on infected cells. d) Proviral DNA was detected by PCR using the genomic DNA of infected cells as templates.

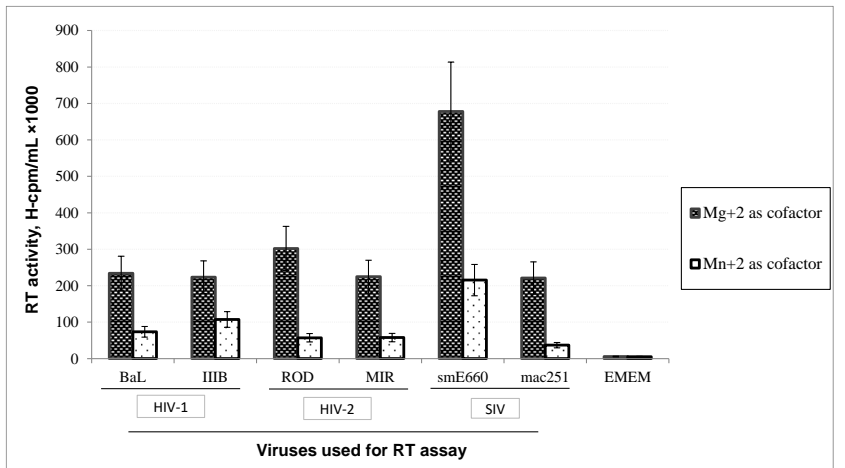

Fig. 2. $\mathrm{Mg}^{2+}$ and $\mathrm{Mn}^{2+}$ dependent Reverse transcriptase (RT) assay. Reverse transcriptase (RT) assay was conducted to detect the indirect concentration of HIV/SIV particles grown in NP-2/CD4/coreceptor cells. RT activities of culture supernatants were measured at the ends of viral propagation when about 80-90 cells became respective viral antigenpositive. Black bars represent $\mathrm{Mg}^{2+-}$ supported RT activities, and white bars exhibit $\mathrm{Mn}^{2+-}$ assisted RT activities.

\section{DISCUSSION}

We investigated comparative techniques of HIV/SIV infection identification in the NP-2/CD4/coreceptor cell line system. We found all the strains of HIV-1, HV-2, and SIV to infection in the cell line conveniently. IFA had identified all the viral infections in the infected cells, which endorsed a gold-standard technique for retroviral infection measurement [33]. Proviral DNA identification in the infected cells had validated IFA observation. This method detects reverse-transcribed HIV DNA integrated into the eukaryotic cellular chromosomes. Syncytia formation is a signature characteristic of retrovirus infection, which was affirmed in this study by identifying MGC. The virion release into cell supernatant after cellular cytopathy had authenticated all the above findings. The method identifies the active lytic phase for the production of infectious virus particles from susceptible eukaryotic host cells.

The RT assay is a popular method for the general detection of various retroviruses. The RT assay carries some 
advantage of detecting HIV or other retroviruses over antigen-based other assays [16], [32]. Some highly sensitive and technically demanding PCR-based RT assays have been developed which can detect as low as three virions per microliter [34]-[36]; however, these were labor-intensive and expensive. Recently, many modified versions of RT assays have been developed that are easy, quick, relatively inexpensive, and convenient to perform [16]. Amplification of HIV-1 virion RNA is presently used for viral load assays, which require infrastructure, facilities for molecular diagnostics, expensive equipment, and skilled technicians, often impractical in resource-limited settings [31]. Measurement of the viral reverse transcriptase (RT) enzyme activity in an alternative to assess the HIV-1 RNA. This low-cost and straightforward viral detection assay would be very useful in resource-limited environments.

In this study, we reported a superior performance of $\mathrm{Mg}^{2+}$ as cofactor over $\mathrm{Mn}^{2+}$ in RT assay for HIV-1, HIV-2, SIV. Similar results were found in an earlier study focusing on RT from primate retroviruses [21]. The reduced RT activity with $\mathrm{Mn}^{2+}$ indicates a mismatching of the cofactor with the enzyme structure. Some earlier studies proposed a stronger affinity of $\mathrm{Mg}^{2+}$ to the cation binding sites of RT acting for the polymerase activity [37]. The study proposed that there are two separate binding sites for $\mathrm{Mg}^{2+}$ and $\mathrm{Mn}^{2+}$ on RT structure bearing unequal catalytic efficiency. Moreover, the binding efficiency of $\mathrm{Mn} 2+$ is more than that of $\mathrm{Mg}^{2+}$; therefore, $\mathrm{Mn}^{2+}$ governs the catalytic activity when both the cations remain present in RT reactions [37]. The further mutational analysis supported the double-binding site model in the polymerase domain of HIV-RT [38].

In summary, the results in the study showed a high level of agreement among different four detection techniques of HIV/SIV infections conducted in the NP-2-based cell line system. With the advantage of low-cost implementation compared with other confirmatory methods, RT assay could be a practical technique in HIV diagnosis programs where molecular biology and serology methods are challenging.

\section{CONCLUSIONS}

$\mathrm{Mg}^{2+}$ remains a better cofactor for $\mathrm{RT}$ assay. $\mathrm{RT}$ is an inexpensive and infrequent methodology to quantify HIV/SIV viral load either in a cell line or human serum. The introduction of this technique would be of great value for resource-limited settings, where the nucleic acid amplification technique (NAAT) or Western blotting is unavailable.

\section{ACKNOWLEDGMENT}

This research was supported by grants-in-aid from the 21st Century COE Program, "Biomedical Research using Accelerated Technology" and Gunma University Graduate School of Medicine.

\section{COMPETING INTERESTS}

None of the authors declared competing or conflict of interest.

\section{AUTHORSHIP CONTRIBUTION}

SI conducted major laboratory experiments and virological assays, prepared the results, and drafted the manuscript. MAM assisted manuscript writing and reviewing. AT assisted microscopy and worked to establish cell lines. HH played vital roles in coordinating the study and helped in manuscript-review.

\section{REFERENCES}

[1] H. zur Hausen, Viruses in human cancers. Eur J Cancer, 1999. 35(14): p. 1878-85.

[2] P.A. Lazo, Structure, DNaseI hypersensitivity and expression of integrated papilloma virus in the genome of HeLa cells. Eur $\mathbf{J}$ Biochem, 1987. 165(2): p. 393-401.

[3] Y. Soda, et al., Establishment of a new system for determination of coreceptor usages of HIV based on the human glioma NP-2 cell line. Biochem Biophys Res Commun, 1999. 258(2): p. 313-21.

[4] A.G. Dalgleish, et al., The CD4 (T4) antigen is an essential component of the receptor for the AIDS retrovirus. Nature, 1984. 312(5996): p. 763-7.

[5] J.S. Allan, J. Strauss, and D.W. Buck, Enhancement of SIV infection with soluble receptor molecules. Science, 1990. 247(4946): p. 1084-8.

[6] H. Choe, et al., The beta-chemokine receptors CCR3 and CCR5 facilitate infection by primary HIV-1 isolates. Cell, 1996. 85(7): p. $1135-48$.

[7] T. Dragic, et al., HIV-1 entry into CD4+ cells is mediated by the chemokine receptor CC-CKR-5. Nature, 1996. 381(6584): p. 667-73.

[8] S. Islam, et al., CKR-L3, a deletion version CCR6-isoform shows coreceptor-activity for limited human and simian immunodeficiency viruses. BMC Infect Dis, 2014. 14: p. 354.

[9] B. Forghani, J.W. Hurst, and C.S. Chan, Advantages of a human immunodeficiency virus type 1 (HIV-1) persistently infected HeLa T4+ cell line for HIV-1 indirect immunofluorescence serology. J Clin Microbiol, 1991. 29(10): p. 2266-72.

[10] R.S. Blumberg, et al., Detection of human T-cell lymphotropic virus type III-related antigens and anti-human T-cell lymphotropic virus type III antibodies by anticomplementary immunofluorescence. J Clin Microbiol, 1986. 23(6): p. 1072-7.

[11] S. Islam, et al., X4-tropic human immunodeficiency virus IIIB utilizes CXCR4 as coreceptor, as distinct from R5X4-tropic viruses. Microbiol Immunol, 2013. 57(6): p. 437-44.

[12] J.D. Lifson, et al., AIDS retrovirus induced cytopathology: giant cell formation and involvement of CD4 antigen. Science, 1986 232(4754): p. 1123-7.

[13] A. Sylwester, et al., HIV-induced T cell syncytia are self-perpetuating and the primary cause of T cell death in culture. J Immunol, 1997. 158(8): p. 3996-4007.

[14] A.B. van 't Wout, H. Schuitemaker, and N.A. Kootstra, Isolation and propagation of HIV-1 on peripheral blood mononuclear cells. Nat Protoc, 2008. 3(3): p. 363-70.

[15] H. Kuno, et al., A simple and rapid reverse transcriptase assay for the detection of retroviruses in cell cultures. Cytotechnology, 1999. 29(3): p. 221-7.

[16] S. Gupta, et al., Can HIV reverse transcriptase activity assay be a lowcost alternative for viral load monitoring in resource-limited settings? BMJ Open, 2016. 6(1): p. e008795

[17] A. Herschhorn and A. Hizi, Retroviral reverse transcriptases. Cell Mol Life Sci, 2010. 67(16): p. 2717-47.

[18] S.P. Goff. Retroviral reverse transcriptase: synthesis, structure, and function. J Acquir Immune Defic Syndr (1988), 1990. 3(8): p. 817-31.

[19] A.G. Filler and A.M. Lever, Effects of cation substitutions on reverse transcriptase and on human immunodeficiency virus production. AIDS Res Hum Retroviruses, 1997. 13(4): p. 291-9.

[20] K.J. Fenstermacher and J.J. DeStefano, Mechanism of HIV reverse transcriptase inhibition by zinc: formation of a highly stable enzyme(primer-template) complex with profoundly diminished catalytic activity. J Biol Chem, 2011. 286(47): p. 40433-42.

[21] J.F. Sears, R. Repaske, and A.S. Khan, Improved Mg2+-based reverse transcriptase assay for detection of primate retroviruses. J Clin Microbiol, 1999. 37(6): p. 1704-8.

[22] Y. Kawakami, et al., Bruton's tyrosine kinase regulates apoptosis and JNK/SAPK kinase activity. Proc Natl Acad Sci U S A, 1997. 94(8): p $3938-42$. 
[23] C. Nishiyama, et al., Overproduction of PU.1 in mast cell progenitors: its effect on monocyte- and mast cell-specific gene expression. Biochem Biophys Res Commun, 2004. 313(3): p. 516-21.

[24] K. Yamaguchi and R.A. Byrn, Clinical isolates of HIV-1 contain few pre-existing proteinase inhibitor resistance-conferring mutations. Biochim Biophys Acta, 1995. 1253(2): p. 136-40.

[25] L. Ratner et al., Complete nucleotide sequences of functional clones of the AIDS virus. AIDS Res Hum Retroviruses, 1987. 3(1): p. 57-69.

[26] N. Shimizu et al., A formylpeptide receptor, FPRL1, acts as an efficient coreceptor for primary isolates of human immunodeficiency virus. Retrovirology, 2008. 5: p. 52.

[27] M. Guyader, et al., Genome organization and transactivation of the human immunodeficiency virus type 2. Nature, 1987. 326(6114): p. 662-9.

[28] Y.M. Naidu et al., Characterization of infectious molecular clones of simian immunodeficiency virus (SIVmac) and human immunodeficiency virus type 2: persistent infection of rhesus monkeys with molecularly cloned SIVmac. J Virol, 1988. 62(12): p. 4691-6.

[29] A. Jinno, et al., Identification of the chemokine receptor TER1/CCR8 expressed in brain-derived cells and $\mathrm{T}$ cells as a new coreceptor for HIV-1 infection. Biochem Biophys Res Commun, 1998. 243(2): p. 497-502.

[30] H. Hoshino, et al., Establishment and characterization of 10 cell lines derived from patients with adult T-cell leukemia. Proc Natl Acad Sci U S A, 1983. 80(19): p. 6061-5.

[31] H.S. Iqbal, et al., Use of an HIV-1 reverse-transcriptase enzymeactivity assay to measure HIV-1 viral load as a potential alternative to nucleic acid-based assay for monitoring antiretroviral therapy in resource-limited settings. J Med Microbiol, 2007. 56(Pt 12): p. 1611 1614.

[32] Poiesz, B.J., et al., Detection and isolation of type C retrovirus particles from fresh and cultured lymphocytes of a patient with cutaneous T-cell lymphoma. Proc Natl Acad Sci U S A, 1980. 77(12): p. 7415-9.

[33] M.K. Kiptoo, S.S. Mpoke, and Z.W. Ng'ang'a, New indirect immunofluorescence assay as a confirmatory test for human immunodeficiency virus type 1. East Afr Med J, 2004. 81(5): p. 2225.

[34] W. Heneine, et al., Detection of reverse transcriptase by a highly sensitive assay in sera from persons infected with human immunodeficiency virus type 1. J Infect Dis, 1995. 171(5): p. 1210-6.

[35] H. Pyra, J. Boni, and J. Schupbach, Ultrasensitive retrovirus detection by a reverse transcriptase assay based on product enhancement. Proc Natl Acad Sci U S A, 1994. 91(4): p. 1544-8.

[36] J. Silver, et al., An RT-PCR assay for the enzyme activity of reverse transcriptase capable of detecting single virions. Nucleic Acids Res, 1993. 21(15): p. 3593-4.

[37] E.C. Bolton, A.S. Mildvan, and J.D. Boeke, Inhibition of reverse transcription in vivo by elevated manganese ion concentration. Mol Cell, 2002. 9(4): p. 879-89.

[38] H. Huang, et al., Structure of a covalently trapped catalytic complex of HIV-1 reverse transcriptase: implications for drug resistance. Science, 1998. 282(5394): p. 1669-75.

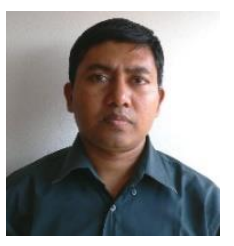

Dr. Salequl Islam was born in a village of Naogaon district, Bangladesh, in 1978. He successfully completed his graduate and M. Sc. in Microbiology from Dhaka University, Bangladesh. Dr. Islam is a current faculty (Associate Professor) at the Department of Microbiology, Jahangirnagar University (JU), Bangladesh. He secured his $\mathrm{PhD}$ from Gunma University School of Medicine in Japan. Mr. Islam began his research career at the Immunology Unit of ICDDR,B in 2003 with Dr Firdausi Qadri. Dr. Islam had transitioned to academia (Jahangirnagar University, JU) soon afterward of his $\mathrm{PhD}$. $\mathrm{He}$ had been awarded a prestigious postdoctoral fellowship sponsored by International AIDS Society and National Institute on Drug Abuse (IAS NIDA 2012). The fellowship had facilitated him to study and work in Johns Hopkins Bloomberg School of Public Health, USA. He worked there in AIDS linked to IntraVenous Experience (ALIVE) cohort at Baltimore, Maryland, in evaluating inflammatory biomarkers among HIV/HCV infected intravenous drug users (IDU). Interfaculty Council For Development Cooperation of KU Leuven (The Katholieke University), Belgium had awarded him for a short visiting research fellowship in 2017. $\mathrm{He}$ worked there at the Virology and Chemotherapy lab at the Rega Institute focusing the discovery of antiviral drugs with their potency, potential toxicity and pharmakokinetics. Dr Islam has been working several years to disseminate American Society for Microbiology (ASM) activities and microbiology research into local students and young researchers. In 2016, ASM had selected him as the head of BioResource Center (BRC) for Bangladesh. $\mathrm{He}$ has participated diverse national and international conferences and presented his research works, notably in: 1) US-Japan Cholera Meeting at Fukuoka, Japan, 2008; 2) AIDS Conference 2012 Washington DC, USA; 3) IAS conference on HIV pathogenesis, Treatment and Prevention 2013, Kuala Lumpur, Malaysia; 4) NIDA International Forum 2016 at Palm Spring, CA, USA; 5) ASM Microbe 2016 at Boston, MA, USA; 6) ASM Biothreat 2018 at Baltimore, MD, USA. 